\title{
NORMAL WEIGHTED COMPOSITION OPERATORS ON THE FOCK SPACE OF $\mathbb{C}^{N}$
}

\author{
LIANKUO ZHAO
}

Abstract. This paper completely characterizes normal weighted composition operators on the Fock space of $\mathbb{C}^{N}$, the form of such operators are expressed explicitly. The characterization of self-adjoint weighted composition operators on the Fock space of $\mathbb{C}^{N}$ is obtained also.

Mathematics subject classification (2010): 47B35.

Keywords and phrases: Fock space, weighted composition operator, bounded, normal, self-adjoint.

\section{REFERENCES}

[1] P. S. Bourdon, S. K. NARAYAn, Normal weighted composition operators on the Hardy space $H^{2}(U)$, J. Math. Anal. Appl. 367 (2010), 278-286.

[2] C. C. Cowen, S. JUnG, E. Ko, Normal and cohyponormal weighted composition operators on $H^{2}$, Operator Theory in Harmonic and Non-commutative Analysis. Springer International Publishing Switzerland, 2014, 69-85.

[3] T. LE, Normal and isometric weighted composition operators on the Fock space, Bull. London. Math. Soc. 46 (2014), 847-856.

[4] T. LE, Self-adjoint, unitary and normal weighted composition operators in several variables, J. Math. Anal. Appl. 395 (2012), 596-607.

[5] L. Li, Y. NAKADA, D. NeSTOR, et al., Normal weighted composition operators on weighted Dirichlet spaces, J. Math. Anal. Appl. 423 (1) (2015), 758-769.

[6] S. UeKI, Weighted composition operator on the Fock space, Proc. Amer. Math. Soc. 135 (5) (2007), $1405-1410$.

[7] S. UeKI, Hilbert-Schmidt weighted composition operator on the Fock space, Int. J. Math. Anal. 1 (2007), 769-774.

[8] L. ZHAO, Unitary weighted composition operaors on Fock space of $\mathbb{C}^{n}$, Complex Anal. Oper. Theory, 8 (2014), 581-590.

[9] L. ZHAO, Invertible weighted composition operators on Fock space of $\mathbb{C}^{N}$, J. Funct. Spaces, (2015), 2015.

[10] L. ZHAO, A class of normal weighted composition operators on the Fock pace of $\mathbb{C}^{n}$, Acta Math. Sin. (English Series), 31 (11) (2015), 1789-1797.

[11] L. ZhaO, C. PANG, A class of weighted composition operators on the Fock pace, J. Math. Res. Appl. 35 (3) (2015), 303-310. 